\title{
Introgression in peripheral populations and colonization shape the genetic structure of the coastal shrub Armeria pungens
}

\author{
R Piñeiro ${ }^{1}$, A Widmer ${ }^{2}$, J Fuertes Aguilar ${ }^{1}$ and G Nieto Feliner ${ }^{1}$ \\ ${ }^{1}$ Real Jardin Botánico, CSIC, Plaza de Murillo 2, Madrid, Spain and ${ }^{2}$ ETH Zurich, Institute of Integrative Biology (IBZ), \\ Universitätstrasse 16, Zurich, Switzerland
}

\begin{abstract}
The coastal shrub Armeria pungens has a disjunct AtlanticMediterranean distribution. The historic range expansion underlying this distribution was investigated using the nuclear internal transcribed spacer region, three plastid regions (namely trnL-F, trnS-fM and matK) and morphometric data. A highly diverse ancestral lineage was identified in southwest Portugal. More recently, two areas have been colonized: (1) Corsica and Sardinia, where disjunct Mediterranean populations have been established as a result of the long-distance dispersal of Portuguese genotypes, and (2) the southern part of the Atlantic range, Gulf of Cadiz, where a distinct lineage showing no genetic differentiation among populations occurs. Genetic consequences of colonization seem to have been more severe in the Gulf of Cadiz than in Corsica-Sardinia. Although significant genetic divergence is
\end{abstract}

associated with low plastid diversity in the Gulf of Cadiz, in Corsica-Sardinia, the loss of plastid haplotypes was not accompanied by divergence from disjunct Portuguese source populations. In addition, in its northernmost and southernmost populations, $A$. pungens exhibited evidence for ancient or ongoing introgression from sympatric congeners. Introgression might have created novel genotypes able to expand beyond the latitudinal margins of the species or, alternatively, these genotypes may be the result of surfing of alleles from other species in demographic equilibrium into peripheral populations of $A$. pungens. Our results highlight the evolutionary significance of genetic drift following the colonization of new areas and the key role of introgression in range expansion.

Heredity (2011) 106, 228-240; doi:10.1038/hdy.2010.46; published online 28 April 2010

Keywords: genetic drift; population bottleneck; colonization; ITS; cpDNA; hybridization

\section{Introduction}

Genetic divergence of populations frequently occurs as a consequence of long periods of allopatry. However, founder effects during the colonization of new areas might also lead to rapid divergence, in addition to diversity loss, associated with random genetic drift in small populations.

The idea that founder effects may promote evolutionary divergence was first proposed by Mayr (1942) in his 'genetic revolution' model of speciation. However, empirical data from a number of documented natural colonization events assessed with molecular markers, such as postglacial range expansions (Tregenza, 2002; Pruett and Winker, 2005; Alsos et al., 2007) and colonizations of islands (Grant et al., 2001; Clegg et al., 2002; Abbott and Double, 2003; Barker et al., 2009) suggest that divergence occurs only after severe founder effects. The severity of a founder effect seems to depend on the dispersal and establishment characteristics that modulate the importance of the genetic drift, in particular (1) the mode of dispersal determines different

Correspondence. Current address: $\mathrm{Dr} R$ Piñeiro, Natural History Museum, University of Oslo, NCB - National Centre for Biosystematics, PO Box 1172 Blindern, NO-0318 Oslo, Norway.

E-mail: rosalia.pineiro@gmail.com

Received 6 April 2009; revised 16 February 2010; accepted 8 March 2010; published online 28 April 2010 patterns of gene flow according to the number of propagules, probability of dispersal based on the availability of vectors and geographical distance; and (2) the success of establishment is directly linked to limiting ecological factors and is driven by natural selection (Baker and Moeed, 1987; Pruett and Winker, 2005).

The role of hybridization in colonization has also been made evident in current and classic evolutionary research by the recurring observation of introgressed genotypes in marginal and/or recently colonized areas in plant and animal studies, including meta-population studies and hybrid zones (Abbott, 1992; Fuertes Aguilar et al., 1999b; Ellstrand and Schierenbeck, 2000; Moody and Les, 2002; Choler et al., 2004; Petit et al., 2004; Albert et al., 2006; Lavergne and Molofsky, 2007; TiéCré et al., 2007).

In this study, we investigate the colonization history of the Atlantic-Mediterranean coastal shrub Armeria pungens (Plumbaginaceae). This species is distributed along a $500 \mathrm{~km}$ coastal belt in southern Atlantic Iberia, from the mouth of the Tagus River to the Gibraltar Strait. It also occurs on two disjunct archipelagos: in the Atlantic, in the Cíes islands (off the Galician coast, Northern Spain), and in the Mediterranean, in Southern Corsica and Northern Sardinia (Figure 1a). The AtlanticMediterranean disjunction was previously assessed with amplified fragment length polymorphisms (AFLPs) and bioclimatic envelope modeling. It was shown to result from long-distance dispersal from the Portuguese Atlantic coast into the Mediterranean islands of Corsica 

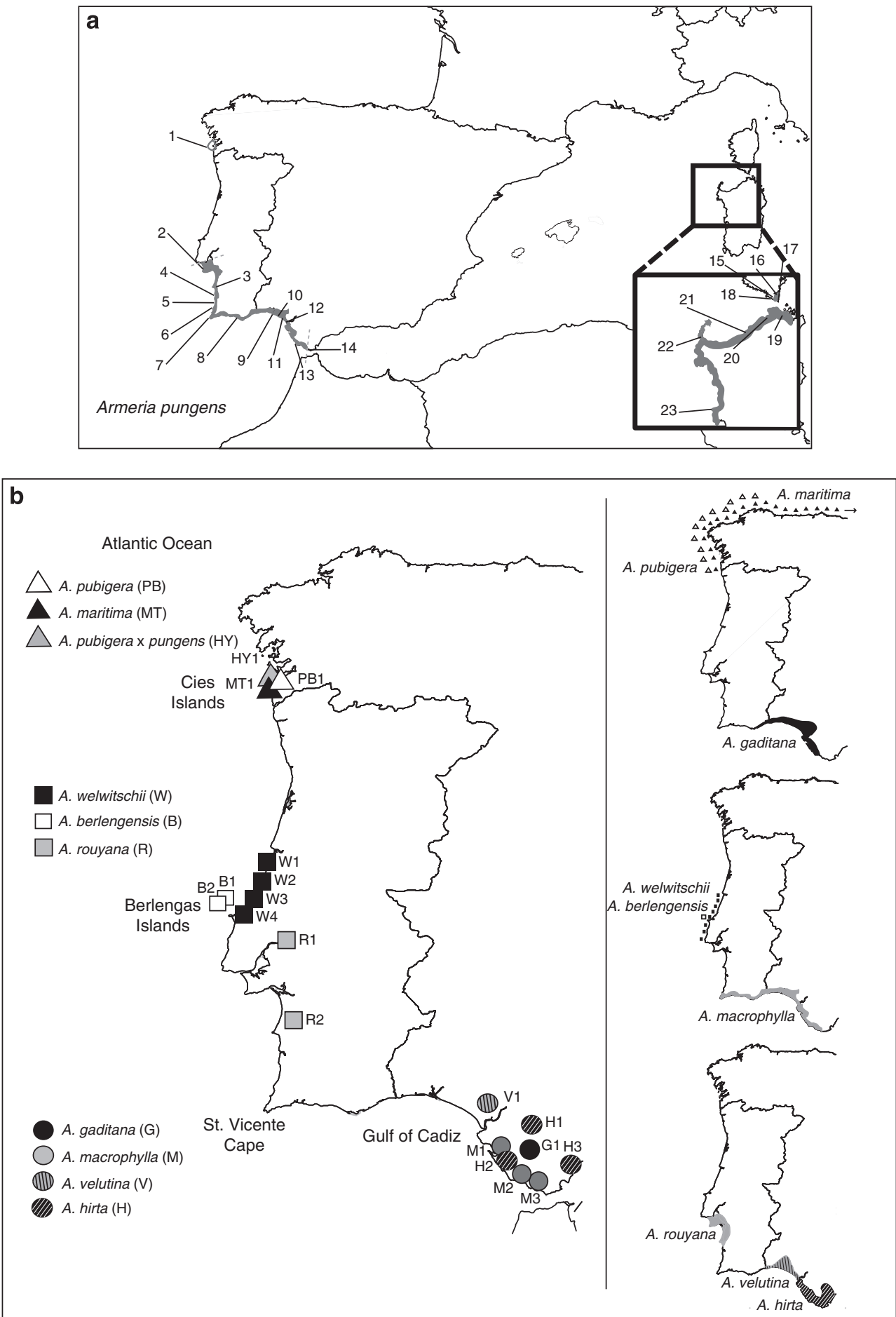

Figure 1 (a) Distribution and sampling of A. pungens. Locations sampled for the morphological and molecular studies (three cpDNA regions trnL-F, trnS-fM matK and nrDNA ITS) are numbered as populations from 1 to 23 , and the total distribution of the species is represented in the background (in gray). (b) Distribution and sampling of the congeners sympatric to A. pungens. A total of 19 populations of coastal and subcoastal Armeria species were sampled for sequencing of three cpDNA regions ( $t r n \mathrm{~L}-\mathrm{F}$, $\operatorname{trnS}$-fM and matK) and partly for the nrDNA ITS. The approximate distribution of the nine species studied is represented according to Bernis (1955); Arrigoni (1970) and Nieto Feliner (1990). Population names and sample information are provided in Supplementary information S1 and S2B.

and Sardinia, probably favored by similar climatic conditions in both areas (Piñeiro et al., 2007).

The populations in the southern part of the Atlantic range (Gulf of Cadiz), which has drier summers than the remaining localities of the species range, were shown to form a distinct and less diverse genetic group, based on
AFLP, despite the absence of physical barriers with Portugal. However, the unordered AFLP data did not allow us to assess whether this distinct group is the result of long-term isolation or recent colonization (Piñeiro et al., 2007). In this study, we address this question using genealogical data, that is, DNA sequences 
that allow identification of the closest relative to the distinct populations in the Gulf of Cadiz. For this purpose, we sequenced three plastid regions-trnL-trnF, $m a t \mathrm{~K}$ and $t r n S-t r n \mathrm{fM}$ - and the nuclear ribosomal internal transcribed spacers (ITSs) across the whole range of A. pungens. We also performed a detailed morphological study. The use of plastid markers is highly suitable for assessing the genetic effects of colonization due to maternal inheritance and low effective population size (Clegg et al., 1994) of the haploid chloroplast genome, which make it more sensitive to genetic drift than the nuclear genome. In agreement with this prediction, patterns of variation of the low-copy nuclear gene GapC in $A$. pungens were shown to be partially blurred by incomplete lineage sorting (Piñeiro et al., 2009). The use of ITS sequences in phylogeography is less straightforward, given that the concerted evolution of different ITS copies usually results in low levels of variation within reproductive groups (Baldwin et al., 1995). In addition, the multicopy nature of these markers and their specific mode of evolution frequently results in a mixture of orthologous and paralogous rDNA copies within a genome, which complicates phylogenetic inference (Álvarez and Wendel, 2003). Nevertheless, we also surveyed this marker, because accumulated data in Armeria (Fuertes Aguilar et al., 1999b; Fuertes Aguilar and Nieto Feliner, 2003; Nieto Feliner et al., 2004) provide a useful background against which new data can be interpreted. Furthermore, following Nieto Feliner and Rosselló (2007), careful use of these markers that includes representative sampling, careful lab protocols and thoughtful analyses can provide useful and even insightful information into species-level evolutionary studies.

Hybridization is very common in natural populations of Armeria owing to weak interspecific reproductive barriers, even between distantly related species, as shown in artificial crossing experiments (Baker, 1966; Lefèbvre, 1974; Nieto Feliner et al., 2001). This process is well documented to affect the structure of populations long after the divergence of species has started (Gutiérrez Larena et al., 2002; Tauleigne-Gomes and Lefèbvre, 2005), and thus needs to be taken into account in any evolutionary study within the genus. In particular, biparentally inherited ITS markers have been useful for documenting reticulate evolution in Armeria, based on the identification of individual additive polymorphisms and a pattern of variation consistent with geography but not with taxonomy (Fuertes Aguilar et al., 1999b). In this study, we addressed the putative incidence of hybridization in the evolution of $A$. pungens through a comparison of ribosomal DNA markers with maternally inherited plastid sequences. The identification of putative maternal donors of introgression events was also attempted through sequencing of plastid markers from congeners sympatric or parapatric to A. pungens (Tauleigne-Gomes and Lefèbvre, 2005).

The specific objectives of this study were as follows:

- To assess whether the proposed long-distance dispersal into Corsica and Sardinia is confirmed with morphological data, plastid DNA and ITS sequences;

- to test for colonization versus isolation in the Gulf of Cadiz;

- to assess the genetic consequences of colonization; and
- to test for introgression from other species of Armeria into $A$. pungens.

\section{Materials and methods}

\section{Study system}

A detailed description of $A$. pungens and its suitability for phylogeographic studies is provided in the study by Piñeiro et al. (2007). The remaining coastal and subcoastal species of Armeria in the Iberian Peninsula are restricted to the western and northern Atlantic coasts (Bernis, 1955; Nieto Feliner, 1990). The western Atlantic coast is the center of diversity for sect. Macrocentron, including Armeria welwitschii, Armeria berlengensis, Armeria pinifolia, Armeria rouyana, Armeria hirta, Armeria gaditana, Armeria macrophylla and Armeria velutina (Figure 1b). In addition, the nonMacrocentron species Armeria pseudoarmeria occurs locally in Cabo da Roca and the surrounding areas. In contrast, the northern Atlantic coast is dominated by the maritima aggr., represented by the widely distributed Armeria maritima and the closely related Iberian endemic Armeria pubigera. The Cíes islands are the only location where sect. Macrocentron and maritima aggr. meet, as A. maritima, A. pubigera and $A$. pungens coexist in the archipelago. The genus Armeria is largely absent from the coasts of Eastern Spain, Southern France and Italy, with the notable exception of Armeria soleirolii in Northern Corsica, as well as A. pungens in Southern Corsica and Northern Sardinia.

\section{Sampling strategy}

For the molecular study, dried leaves and seeds were collected from 23 populations spanning the entire range of the species (Figure 1a; Supplementary information S1). A distance of $10 \mathrm{~m}$ between plants was maintained. Seeds were germinated after a cold treatment of 1 month, and seedlings were cultivated under greenhouse conditions. The average sampling size was two individuals per population for the ITS region $(N=59)$ and five individuals per population for the three plastid regions $(N=112)$. For the morphological study, voucher specimens were collected for 21 of the populations and deposited in the herbarium of the Real Jardín Botánico (MA). The average sampling size was 13 individuals per population $(N=241)$.

To test for the origin of the divergent plastid haplotypes in populations from the margins of the distribution of $A$. pungens, an extended interspecific sampling of coastal and subcoastal species of Armeria was undertaken. To examine the northernmost disjunct population, we sampled all congeners from the Cíes islands ( $A$. maritima, A. pubigera and putative hybrids between $A$. pungens and $A$. pubigera), and three of four species from the central portion of the Iberian Atlantic coast ( $A$. welwitschii, $A$. berlengensis and $A$. rouyana). In the vicinity of the southernmost population, all extant subcoastal species ( $A$. hirta, A. velutina, A. macrophylla and $A$. gaditana) were studied. The locally distributed species $A$. pseudoarmeria and the sublittoral species A. pinifolia were the only coastal species from Atlantic Iberia not included in the study. Therefore, in addition to the samples of A. pungens, a total of 37 individuals from 19 populations belonging to 10 taxa were included (Figure 1b; Supplementary information S2). 
DNA isolation, PCR amplification and sequencing DNA isolation from dried or fresh leaves was performed using the DNeasy Plant Mini Kit (Qiagen, Valencia, CA, USA). The ITS region (ITS1, ITS2 and the 5.8S gene) was amplified and sequenced with universal primers P1A and P4 (Fuertes Aguilar et al., 1999b). The trnL-trnF intergenic spacer was amplified with primers e and $\mathrm{f}$ (Taberlet et al., 1991) and sequenced with primer e. The trnS-fM intergenic spacer was amplified with universal primers trnS and trnfM (Demesure et al., 1995) or, in two fragments, using primer combinations trnS-intM and intS-trnfM for amplification and trnS and intS for sequencing. The matK-encoding gene was amplified and sequenced with specific primers intK2 and intK3. For recalcitrant samples, PCR products obtained with primers extK2 and extK3 were used for nested amplification with intK2 and intK3. Specific primers intM, intS, extK2, extK3, int2 and intK3 (see Supplementary information S3) were designed using the Primer3 software (Rozen and Skaletsky, 2000). PCR reactions contained $20-200 \mathrm{ng}$ of template DNA, $10 \times$ or $5 \times$ PCR buffer, $1.5 \mathrm{mM} \mathrm{MgCl} 2,1 \mathrm{mM}$ dNTPs, $0.4 \mu \mathrm{M}$ of each primer and $0.4 \mu \mathrm{l}$ Taq (Promega, Madison, WI, USA), or were performed with Ready-To-Go beads (Amersham Biosciences Europe $\mathrm{GmbH}$, Cerdanyola, Barcelona, Spain) with 10-500 ng template DNA, $0.2-0.8 \mathrm{mM}$ of each primer and $4 \%$ dimethyl sulfoxide or bovine serum albumin. The PCR conditions for ITS followed those described in the study by Lihová et al. (2004), with a $52-54{ }^{\circ} \mathrm{C}$ annealing temperature. The trnL-F profile followed the procedure of Gutiérrez Larena et al. (2002), with a $50{ }^{\circ} \mathrm{C}$ annealing temperature. The trnS-fM and trnK-K profiles are reported in Supplementary information S4. Sequencing reactions were run on an ABI Prism sequencer 377 or 3730 (Applied Biosystems, Foster City, CA, USA).

\section{Armeria pungens}

cpDNA: Three plastid regions-trnL-F, trnS-fM and matK-were chosen. A total of 112 concatenated sequences of $A$. pungens were obtained and manually aligned with Bioedit 5.0.9 (Hall, 1999) following the guidelines proposed by Kelchner (2000). The same individuals were sampled for matK and trnS-fM, whereas different individuals from the same populations were included for trnL-F in 69 cases. The three plastid regions were analyzed together in the same network after the finding that 19 of 23 populations were monomorphic for trnL-F. To control for putative artificial consensus sequences in the four populations found to be polymorphic for trnL-F, we checked two cases (populations 3 and 23), in which concatenations of different individuals produced singletons (haplotypes $\mathrm{L}$ and $\mathrm{D})$. These two haplotypes were removed from an alternative analysis without producing any significant difference. Genealogical relationships among haplotypes were estimated in a statistical parsimony network using TCS 1.21 (Clement et al., 2000). For mononucleotide repeats of different lengths, each contiguous gap position was coded as a single substitution. Indels were coded as a single mutation.

Population genetic parameters were calculated using dnaSP 4.10.7 (Rozas et al., 2003) and Haplonst (Pons and Petit, 1996). The within-population diversity was estimated first in terms of allele frequencies with Nei's haplotypic diversity, $\mathrm{Hd}$, and second in terms of the weighted sequence divergence with the nucleotide diversity, $\pi$, the average number of nucleotide differences per site between two sequences (Rozas et al., 2003). Population differentiation was estimated for different geographical regions based on haplotype frequencies only, $\mathrm{G}_{\mathrm{st}}$, and both on haplotype frequencies and genetic distance between haplotypes, $\mathrm{N}_{\mathrm{st}}$ (Pons and Petit, 1996). The difference between the two estimates was assessed with a haplotype identity permutation test (10000 permutations) implemented in PERMUT 1.0 (http:// www.pierroton.inra.fr). The regional allelic richness, A, corrected for sample size $(N=28)$ using the rarefaction method of El Mousadik and Petit (1996), was calculated using FSTAT 2.9.3.2 (Goudet, 2001).

Internal transcribed spacers: A total of 59 ITS consensus sequences of $A$. pungens were generated from forward and reverse sequences and aligned with Bioedit 5.0.9. Intraindividual polymorphisms, detected as double peaks in both the forward and reverse electropherograms, were carefully checked and coded using ambiguity codes of the IUPAC (The International Union of Pure and Applied Chemistry) according to Fuertes Aguilar et al. (1999a).

Morphometrics: In all, 19 morphological variables (15 quantitative continuous and 4 qualitative, Supplementary information S5A and B) were recorded. We performed a principal components analysis based on a correlation matrix of the quantitative characters and four derived ratios (Supplementary information S5A) with SPSS 14 for Windows (SPSS Inc.). To maximize independence of characters, one original measure of each ratio was removed. Qualitative characters (Supplementary information S5B) were examined by calculating the percentage of individuals exhibiting each character state per population.

\section{Sympatric congeners}

cpDNA: A chloroplast DNA (cpDNA) matrix containing 71 sequences was constructed by adding 36 Armeria sp. sequences to a reduced matrix of 34 sequences of $A$. pungens (selected out of 112 by deleting identical sequences of each population). One specimen of Psylliostachys suworowi was used as the outgroup following the study by Lledó et al. (1998). Gaps resulting from alignment were treated as missing values.

Phylogenetic analyses were performed using Maximum Parsimony and Bayesian approaches. Maximum Parsimony was carried out using PAUP v. $4.0 \mathrm{~b} 10$ (Swofford, 2002) under Fitch parsimony through heuristic searches with 100 random taxon addition replicates, holding 100 trees at each step and TBR branch swapping on all trees (options steepest descent off and multrees off). Branch support was calculated using the fast bootstrap option with 10000 replicates under two different treatments of indels: by simply treating gaps resulting from alignment as missing values and by adding an additional binary character representing the presence/absence of the indel. Before the Bayesian analyses, the cpDNA matrix for each plastid region was imported into Modeltest 1.1b (Nylander, 2003) to select the model of evolution under the Akaike information criterion. Subsequently, a Bayesian analysis was run 
with Mr Bayes 3.1 (Ronquist and Huelsenbeck, 2003), setting three parallel runs for five million generations. Convergence was diagnosed using the s.d. of split frequencies. A total of 1250000 trees (25\%) were discarded and the remaining trees were compiled into a majority rule consensus tree, using the posterior probability as a measure of clade support.

Internal transcribed spacers: ITS sequences of $A$. pungens were reanalyzed within the framework of a previous ITS phylogenetic study of the genus Armeria representing $70 \%$ of the species (Fuertes Aguilar and Nieto Feliner, 2003). In that study, most coastal Iberian species of sect. Macrocentron were shown to harbor a set of closely related ITS ribotypes falling within the same clade. For simplicity, the whole clade will be referred to here as ribotype $\mathrm{R} 1$. This ribotype was also detected in many inland western Iberian species. In contrast, coastal species from the maritima aggr. formed an independent clade that will be referred to as ribotype R7. Finally, the Corso-Sardinian species, Armeria leucocephala, Armeria multiceps, A. soleirolii and Armeria sardoa, shared a single ITS ribotype (ribotype R5) remarkably distant from ribotype R1.

In populations in which $A$. pungens is known to be sympatric with congeners from other ITS clades, individuals were checked for putative polymorphic sites revealing hybridization (Fuertes Aguilar and Nieto Feliner, 2003; Keller et al., 2008). In particular, additive polymorphic sites revealing the coexistence of ribotype R1 (characteristic of $A$. pungens) and ribotype R7 (characteristic of the A. maritima clade) were checked in the Cíes Islands, whereas in Corsica and Sardinia, additive polymorphic sites between ribotypes R1 and R5 were sought. For this purpose, 10 additional sequences from congeners $A$. pubigera, A. maritima, A. pungens $\times A$. pubigera, and $A$. macrophylla were obtained.

\section{Results}

Molecular and morphological variation within $A$. pungens The sequencing of $t r n \mathrm{~L}-\mathrm{F}, \operatorname{trn} \mathrm{S}-\mathrm{fM}$ and matK in A. pungens resulted in a final matrix of 112 individuals and $3037 \mathrm{bp}$. In all, 20 nucleotide substitutions, 3 mononucleotide repeats and one 6-bp indel defined 16 haplotypes (Supplementary information S6). Identical networks, only showing differences in branch lengths, were produced by adding or excluding the presence/absence characters. The geographic distribution of haplotypes and relationships among them are represented in Figures $2 \mathrm{a}$ and $\mathrm{b}$.

The ITS matrix for A. pungens encompassed 59 individuals and $629 \mathrm{bp}$. A. pungens presented only ITS sequences corresponding to ribotype R1, also present in many western Iberian species (Fuertes Aguilar and Nieto Feliner, 2003), across its whole range, even in Corsica and Sardinia (Table 1). However, five within-individual polymorphic sites were detected. On the basis of our previous knowledge of the variation of this marker in Armeria, these polymorphic sites are interpreted as the intragenomic occurrence of more than one ITS sequence (Fuertes Aguilar et al., 1999a; Nieto Feliner and Rosselló, 2007). Therefore, populations that share the same polymorphic sites are interpreted as having the same additional ITS sequence besides R1. Accordingly, the five ITS profiles recorded through direct sequencing are named R1a, R1b, R1c, R1d and R1e (Table 1; Figure 2c), meaning that the R1a profile contains only R1, whereas the other four contain different additional ITS sequences. Some of the least frequent ITS copies may not have been sequenced, given that the amplified products were not cloned. Nonetheless, the low levels of ITS variation observed through direct sequencing discouraged us from a more thorough sampling of the ITS variation.

The first three principal components extracted in the principal components analysis of quantitative morphological variables explained $68.89 \%$ of the overall variance (Supplementary information S5A). The scatterplot of 241 individuals against PC1 and PC2 (Supplementary information S7) showed no morphometric discontinuities, revealing the absence of morphological differentiation of A. pungens across geographical areas.

\section{The main genetic and morphological groups within}

\section{A. pungens}

cpDNA and ITS markers both revealed two main genetic groups comprising most of the populations within A. pungens: the 'Portuguese-Corso-Sardinian group' (populations 2-7 and 15-23) and the 'Gulf of Cadiz lineage' (populations 8-13). The two highly divergent haplotypes corresponding to populations on the margins of the range of $A$. pungens (populations 1 and 14) are treated below.

The Portuguese and Corso-Sardinian areas shared three plastid haplotypes (namely A, C and G; Figures $2 a$ and b) and two ITS profiles (the standard one, R1a, and R1b) (Figure 2c; Table 1). Portuguese populations (populations 2-7) harbored the highest number of plastid DNA variants within the species, 10 of 16 (haplotypes A, B, C, F, G, H, I, J, K and L; Figures 2a and b). In contrast, Corso-Sardinian populations (populations 15-23) had five plastid haplotypes: a subset of haplotypes from Portugal (the dominant haplotype $\mathrm{A}$ and the local haplotypes $\mathrm{C}$ and $\mathrm{G}$ ) and two exclusive local haplotypes (haplotype $\mathrm{M}$ in population 15 and haplotype $\mathrm{D}$ in population 23; Figures 2a and b). The Gulf of Cadiz lineage harbored an inferred ancestral haplotype in one individual (population 8, haplotype E), closely related to Southern Portugal haplotypes (B, G and $\mathrm{H}$ ) and the exclusive haplotype $\mathrm{N}$, present in all six populations, derived from the same lineage but separated by several mutational steps (Figures 2a and b). Two ITS profiles (R1c and R1d; Figure 2c; Table 1) were also exclusive to this lineage. Nonetheless, the standard ITS profile appeared in a single population and was shared with the Corso-Sardinian and southern Portuguese populations (R1a; Figure 2c; Table 1).

The nature of the ITS variation detected in A. pungens, involving the coexistence of more than one ITS repeat within individuals, did not allow for estimations of population diversity parameters. Genetic diversity and differentiation were thus calculated based only on plastid markers. The overall level of plastid differentiation in A. pungens was high with $\mathrm{G}_{\mathrm{st}}=0.73$ (average for angiosperms: $\mathrm{G}_{\mathrm{st}}=0.63$; Petit et al., 2005). Portuguese populations exhibited high intrapopulation (average $h_{s}=0.48$; Table 2) and regional $\left(A=10 ; h_{t}=0.90 ;\right.$ Table 2) 

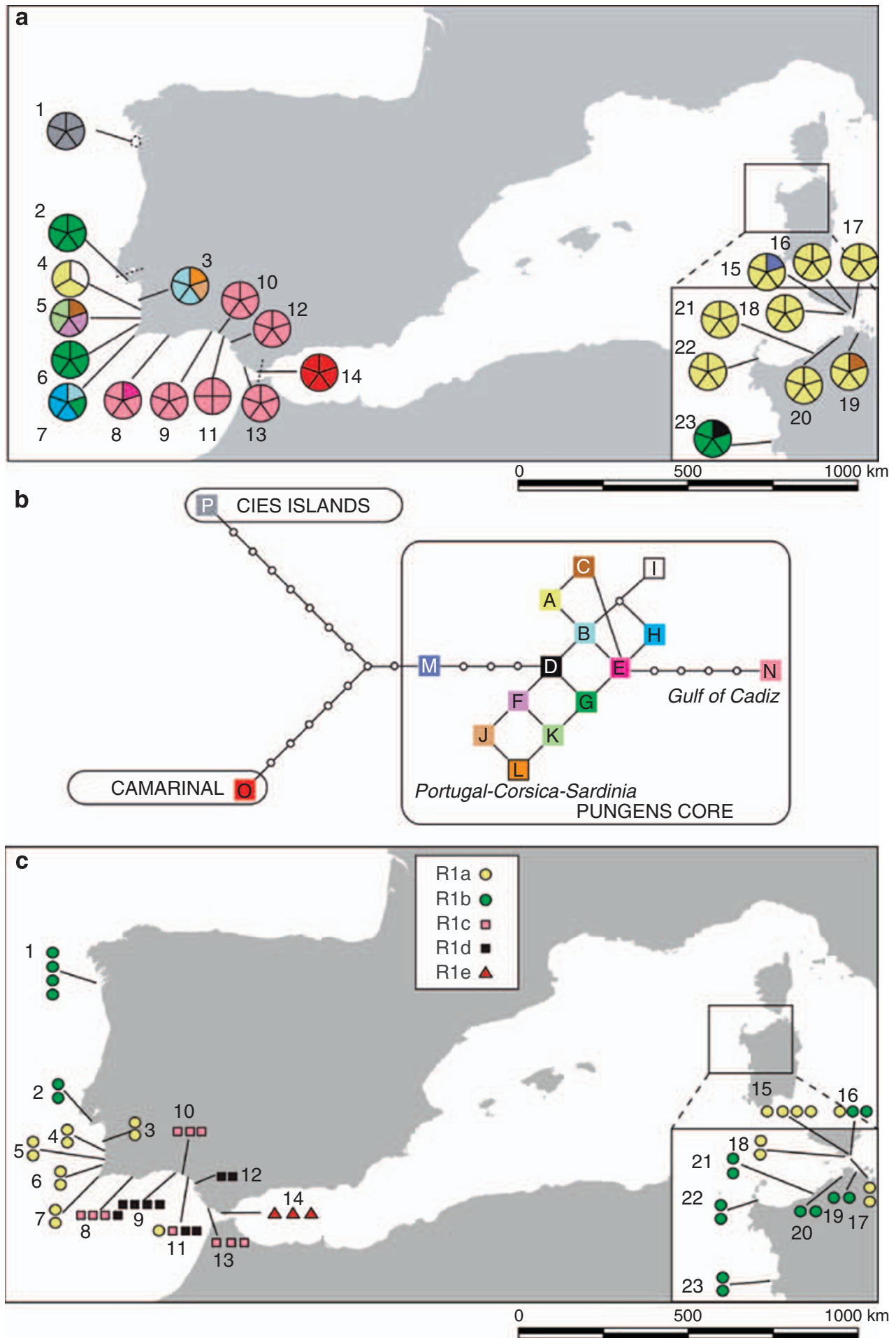

Figure 2 Main genetic groups in A. pungens. (a) Geographic distribution of the 16 plastid haplotypes (trnL-F, trnS-fM, matK) found in 112 individuals from 23 populations of A. pungens (numbered as in Supplementary information S1). (b) Statistical parsimony network showing the genealogical relationships among the 16 plastid haplotypes. (c) Geographic distribution of ITS profiles across 59 individuals from 23 populations of A. pungens: R1a denotes typical Western Mediterranean ribotype (Fuertes Aguilar and Nieto Feliner, 2003), without polymorphic sites in direct sequencing; R1b, R1c, R1d, R1e indicate polymorphic site in different positions that are interpreted as intragenomic cooccurrence of more that one ITS sequence (Table 1).

diversities, resulting in a moderate level of regional differentiation $\left(\mathrm{G}_{\mathrm{st}}=0.47\right.$; Table 2$)$. The haplotype diversity within populations was high in populations $3,4,5$ and 7 ( $\mathrm{Hd}=0.67-0.80$; Table 3 ), whereas populations 2 and 6 were fixed for a single haplotype $(\mathrm{Hd}=0$;
Table 3). The diversity in Corsica-Sardinia was moderate $\left(h_{s}=0.09 ; \mathrm{Hd}=0 ; h_{t}=0.26 ; A=4\right)$, also resulting in moderate regional differentiation $\left(\mathrm{G}_{\mathrm{st}}=0.66\right.$; Table 2$)$. Finally, the genetic variation was almost zero along the Gulf of Cadiz ( $\mathrm{Hd}=0.07 ; A=2 ; h_{t}=0 ; \mathrm{G}_{\mathrm{st}}=0$; Tables 2 
Table 1 Nucleotide variation across the aligned ITS1-5.8S-ITS2 sequences found in Armeria pungens populations

\begin{tabular}{|c|c|c|c|c|c|c|c|c|c|c|c|}
\hline & 62 & 99 & 380 & 381 & 390 & 391 & 392 & 416 & 417 & 448 & 520 \\
\hline \multicolumn{12}{|c|}{ Armeria spp. (Fuertes Aguilar and Nieto Feliner, 2003) } \\
\hline Western Iberian clade R1 & $\mathrm{T}$ & C & $\mathrm{T}$ & $\mathrm{C}$ & $\mathrm{C}$ & $\mathrm{T}$ & $\mathrm{T}$ & G & G & $\mathrm{C}$ & $\mathrm{A}$ \\
\hline Corsica-Sardinia clade R5a & . & . & . & & 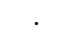 & $\mathrm{C}$ & $\mathrm{C}$ & $\mathrm{T}$ & $\mathrm{T}$ & . & . \\
\hline A. maritima-alpina clade R7 & . & . & . & $\mathrm{T}$ & $\mathrm{T}$ & C & C & . & . & . & G \\
\hline \multicolumn{12}{|l|}{ Armeria pungens $(N=59)$} \\
\hline R1a & $\mathrm{T}$ & C & $\mathrm{T}$ & $\mathrm{C}$ & $\mathrm{C}$ & $\mathrm{T}$ & $\mathrm{T}$ & G & G & $\mathrm{C}$ & A \\
\hline $\mathrm{R} 1 \mathrm{~b}$ & . & $\mathrm{Y}$ & . & . & . & . & . & . & . & . & \\
\hline R1c & $\mathrm{Y}$ & . & . & . & . & . & $\mathrm{Y}$ & . & . & . & \\
\hline R1d & $\mathrm{Y}$ & . & 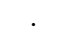 & . & . & . & . & . & . &  & \\
\hline R1e & $\cdot$ & $\cdot$ & Y & . & . & . & . & . & . & Y & \\
\hline \multicolumn{12}{|c|}{ Putative hybrids or introgressed individuals from sympatric populations $(N=10)$} \\
\hline A. pungens $\times A$. pubigera (Cíes) & . & $\mathrm{Y}$ & . & $\mathrm{Y}$ & $\mathrm{Y}$ & $\mathrm{Y}$ & $\mathrm{Y}$ & . & . & . & $\mathrm{R}$ \\
\hline A. maritima, A. pubigera (Cíes) & . & . & . & $\mathrm{Y}$ & $\mathrm{Y}$ & $\mathrm{Y}$ & $\mathrm{Y}$ & . & . & 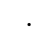 & $\mathrm{R}$ \\
\hline A. macrophylla (Camarinal) & . & . & Y & & . & . & . & . & . & Y & 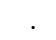 \\
\hline
\end{tabular}

Abbreviations: IUPAC, The International Union of Pure and Applied Chemistry; pop., population; ITS, internal transcribed spacer. Clade nomenclature for reference purposes follow Fuertes Aguilar and Nieto Feliner, 2003. The five ITS profiles recorded through direct sequencing in A. pungens are named R1a, R1b, R1c, R1d and R1e, meaning that the R1a profile only contains R1, whereas the other four contain different additional ITS sequences. Within-individual additive sites are coded according to the IUPAC code. Nucleotides identical to the top sequences are indicated by dots.

Table 2 Regional cpDNA diversity and differentiation (trnL-F, trnS-fM, matK) in Armeria pungens

\begin{tabular}{lccccccccc}
\hline Region & $\begin{array}{c}\mathrm{N} \text { individuals } \\
\text { (N pops. })\end{array}$ & $\begin{array}{c}A \\
(\mathrm{~N}=28)\end{array}$ & $\mathrm{h}_{\mathrm{s}}$ & $\mathrm{h}_{\mathrm{t}}$ & $G_{s t}$ & $v_{s}$ & $v_{t}$ & $N_{s t}$ & $N_{s t}$ perm \\
\hline Portugal & $28(6)$ & 10 & $0.48(0.1522)$ & $0.90(0.1131)$ & $0.47(0.1066)$ & $0.47(0.1561)$ & $0.90(0.1668)$ & $0.48(0.0701)$ & 0.45 \\
Gulf of Cadiz & $29(6)$ & 2 & 0 & 0 & 0 & 0 & 0 & 0 \\
Corsica-Sardinia & $45(9)$ & 4 & $0.09(0.0588)$ & $0.26(0.1751)$ & $0.66(0.0648)$ & $0.04(0.0238)$ & $0.27(0.1947)$ & $0.86(0.0452)$ & $0.65^{\mathrm{a}}$ \\
\hline
\end{tabular}

Abbreviations: A, allelic richness corrected for sample size $(N=28)$; cpDNA, chloroplast DNA; $\mathrm{G}_{s t}$, genetic differentiation based on haplotype frequencies only; $h_{s}$, average within-population diversity based on haplotype frequencies only; $h_{t}$, total genetic diversity based on haplotype frequencies only; $N$, sampling size; $N_{\text {st }}$, genetic differentiation based on haplotype frequencies and genetic distance between haplotypes; $\mathrm{N}_{\mathrm{st}}$ perm, permuted $\mathrm{N}_{\mathrm{st}}$ based on permutated distances between pairs of haplotypes; pop., population; $\mathrm{v}_{\mathrm{s}}$, average within-population diversity based on haplotype frequencies and genetic distance between haplotypes; $v_{t}$, total genetic diversity based on haplotype frequencies and genetic distance between haplotypes.

s.d. is indicated in parentheses.

${ }^{\mathrm{a}} P<0.01$.

and 3). The estimate of the overall genetic differentiation among A. pungens populations that takes into account allele similarity was significantly higher $\left(\mathrm{N}_{\mathrm{st}}=0.85\right)$ than the estimate based on allele frequencies (confirmed by the permutation of haplotype identities test, $U=\mathrm{G}_{\mathrm{st}}$ / $\mathrm{N}_{\text {st }}=2.15 ; \mathrm{N}_{\text {st }}$ perm $=0.70$ with $P<0.01$; Pons and Petit, 1996). In Portugal, there was almost no difference between $\mathrm{G}_{\mathrm{st}}(0.47)$ and $\mathrm{N}_{\mathrm{st}}$ (0.48; Table 2), indicating mutation-drift equilibrium. In Corsica-Sardinia, $\mathrm{N}_{\mathrm{st}}$ (0.86) was also significantly higher than $\mathrm{G}_{\mathrm{st}}(0.66$; Table 2$)$.

In contrast to the principal components analysis based on quantitative characters, the leaf pubescence showed a clear geographical trend that is consistent with the main genetic groups (Figure 3). Most plants in populations along the Gulf of Cadiz displayed glabrous to subglabrous leaves, whereas those in Portugal-Corsica-Sardinia exhibited mostly ciliate leaves on middle veins. An exception is Bordeira (population 6), with dense pubescence on leaves and scapes.

\section{Molecular variation in sympatric congeners}

The cpDNA matrix including the sympatric species had $3057 \mathrm{bp}$ and 94 variable sites, 23 of them parsimony informative. The majority rule consensus of the 87 trees retained in the parsimony analysis was constructed. For the Bayesian analyses, the data were partitioned according to the model of evolution of each plastid marker revealed by Modeltest 1.1b: F81 + I for trnL-F, HKY for SfM and GTR + I for matK. The topology of the majority rule consensus tree was almost identical to that of the parsimony tree. Two weakly supported main clades, $\mathrm{A}$ and B, were present (Figure 4). Clade A included two subclades (labeled 1 and 2) and eight single terminals. The relationships between clades 1 and 2 were not resolved. Clade 1 (60\% bootstrap; 99 posterior probability) contained the majority of $A$. pungens sequences, thus representing the $A$. pungens core lineage. Within this core, the Gulf of Cadiz lineage, comprising populations 8-13 (subclade 1a; 85\% bootstrap; 100 posterior probability), was clearly differentiated from the populations in Portugal-Corsica-Sardinia, comprising populations 2-7 and 15-23 and one individual from population 8 (haplotype E). Populations $\mathrm{RO} 1$ and $\mathrm{RO} 3$ (A. rouyana), V1 (A. velutina) and M1 (A. macrophylla), as well as individuals from G1 (A. gaditana) and W1 (A. welwitschii), also fell close to the Portuguese-Corso-Sardinian samples. Clade 2 (70\% bootstrap; 99 posterior probability) was restricted to the southern Armeria species, 
including $A$. pungens from Camarinal (population 14), $A$. macrophylla, $A$. hirta and A. gaditana. The close relationship between the Camarinal haplotype and its sympatric population of $A$. macrophylla had some support

Table 3 Within-population cpDNA diversity (trnL-F, trnS-fM, matK) in Armeria pungens

\begin{tabular}{|c|c|c|c|}
\hline Population & $\mathrm{N}$ & $\mathrm{Hd}$ & $\pi \times 10^{-}$ \\
\hline \multicolumn{4}{|l|}{ Cies Islands } \\
\hline Pop. 1 & 5 & 0.00 & 0.00 \\
\hline \multicolumn{4}{|l|}{ Portugal } \\
\hline Pop. 2 & 5 & 0.00 & 0.00 \\
\hline Pop. 3 & 5 & 0.70 & 1.01 \\
\hline Pop. 4 & 3 & 0.67 & 0.48 \\
\hline Pop. 5 & 5 & 0.80 & 0.51 \\
\hline Pop. 6 & 5 & 0.00 & 0.00 \\
\hline Pop. 7 & 5 & 0.70 & 0.29 \\
\hline \multicolumn{4}{|l|}{ Gulf of Cadiz } \\
\hline Pop. 8 & 5 & 0.40 & 0.29 \\
\hline Pop. 9 & 5 & 0.00 & 0.00 \\
\hline Pop. 10 & 5 & 0.00 & 0.00 \\
\hline Pop. 11 & 4 & 0.00 & 0.00 \\
\hline Pop. 12 & 5 & 0.00 & 0.00 \\
\hline Pop. 13 & 5 & 0.00 & 0.00 \\
\hline \multicolumn{4}{|l|}{ Camarinal } \\
\hline Pop. 14 & 5 & 0.00 & 0.00 \\
\hline \multicolumn{4}{|c|}{ Corsica-Sardinia } \\
\hline Pop. 15 & 5 & 0.40 & \\
\hline Pop. 16 & 5 & 0.00 & 0.58 \\
\hline Pop. 17 & 5 & 0.00 & 0.00 \\
\hline Pop. 18 & 5 & 0.00 & 0.00 \\
\hline Pop. 19 & 5 & 0.40 & 0.00 \\
\hline Pop. 20 & 5 & 0.00 & 0.14 \\
\hline Pop. 21 & 5 & 0.00 & 0.00 \\
\hline Pop. 22 & 5 & 0.00 & 0.00 \\
\hline Pop. 23 & 5 & 0.40 & 0.00 \\
\hline Total range & 112 & 0.19 & 0.14 \\
\hline
\end{tabular}

Abbreviations: cpDNA, chloroplast DNA; $H d$, haplotype diversity; $N$, sample size; $\pi$, nucleotide diversity; pop., population.
(63\% bootstrap; 100 posterior probability). Clade B (50\% bootstrap; 70 posterior probability) encompassed $A$. pungens from the Cíes islands, two putative hybrids $A$. pungens $\times$ A. pubigera, A berlengensis (populations B1, B2) and $A$. welwitschii (populations W1, W2, W3, W4). The congeners from Cíes (A. pubigera and A. maritima) fell apart from the Cíes samples of $A$. pungens as single terminals in a polytomy with the two main clades together with the remaining three putative hybrids, $A$. pungens $\times A$. pubigera. Bootstrap supports and posterior probabilities of the major clades decreased when additional binary characters were added to represent the presence/absence of indels, which indicates that indels are homoplastic above the species level.

In the Cíes islands, additive patterns were found in ITSs between the R1b profile of $A$. pungens and the R7 in the putative hybrids, $A$. pungens $\times A$. pubigera. A. maritima and $A$. pubigera also presented the same pattern (Table 1). In Camarinal, $A$. pungens exhibited the R1e profile shared with the sympatric species $A$. macrophylla. Finally, in Corsica and Sardinia, A. pungens showed the western Mediterranean ribotype R1 profile (that is, the standard R1a profile) without additions with respect to the ribotype of the other Corso-Sardinian species (R5).

\section{The genetic divergence of peripheral populations of A. pungens}

The southernmost population and the disjunct northernmost population of $A$. pungens were found to be significantly different from the two main genetic groups. The southernmost population (population 14-Camarinal) displayed two ITS polymorphic sites at positions 380 and 448 (profile R1e: Table 1; Figure 2c), which were lacking in the remaining populations, together with an exclusive divergent plastid haplotype (O; Figures 2a and b) and puberulous leaves (Figure 3) that were also lacking elsewhere within A. pungens. In the northernmost island population (population 1-Cíes), conflict between the maternally inherited plastid sequences and

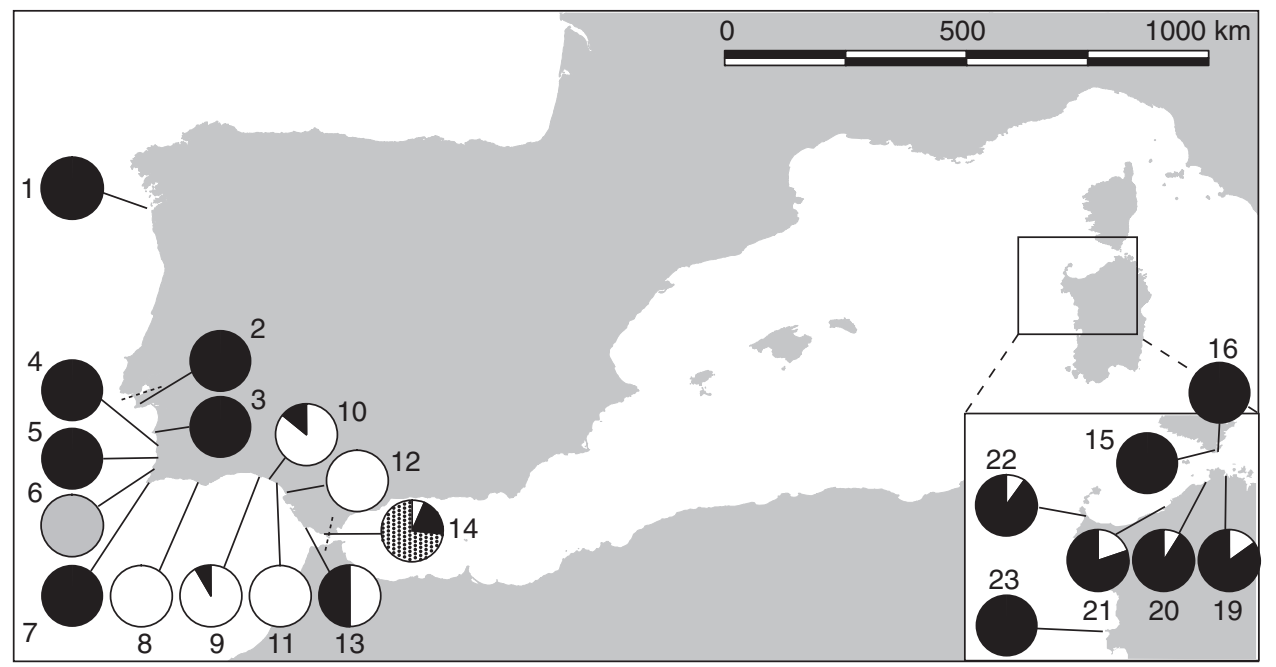

Figure 3 Geographic distribution of the leaf pubescence in A. pungens. The percentages of ciliate (black), glabrous (white), dense pubescent (gray) and puberulous (patterned) leaves are indicated for each population. A. pungens individuals are labeled with population numbers as described in Supplementary information S1 and Figure 2a. 


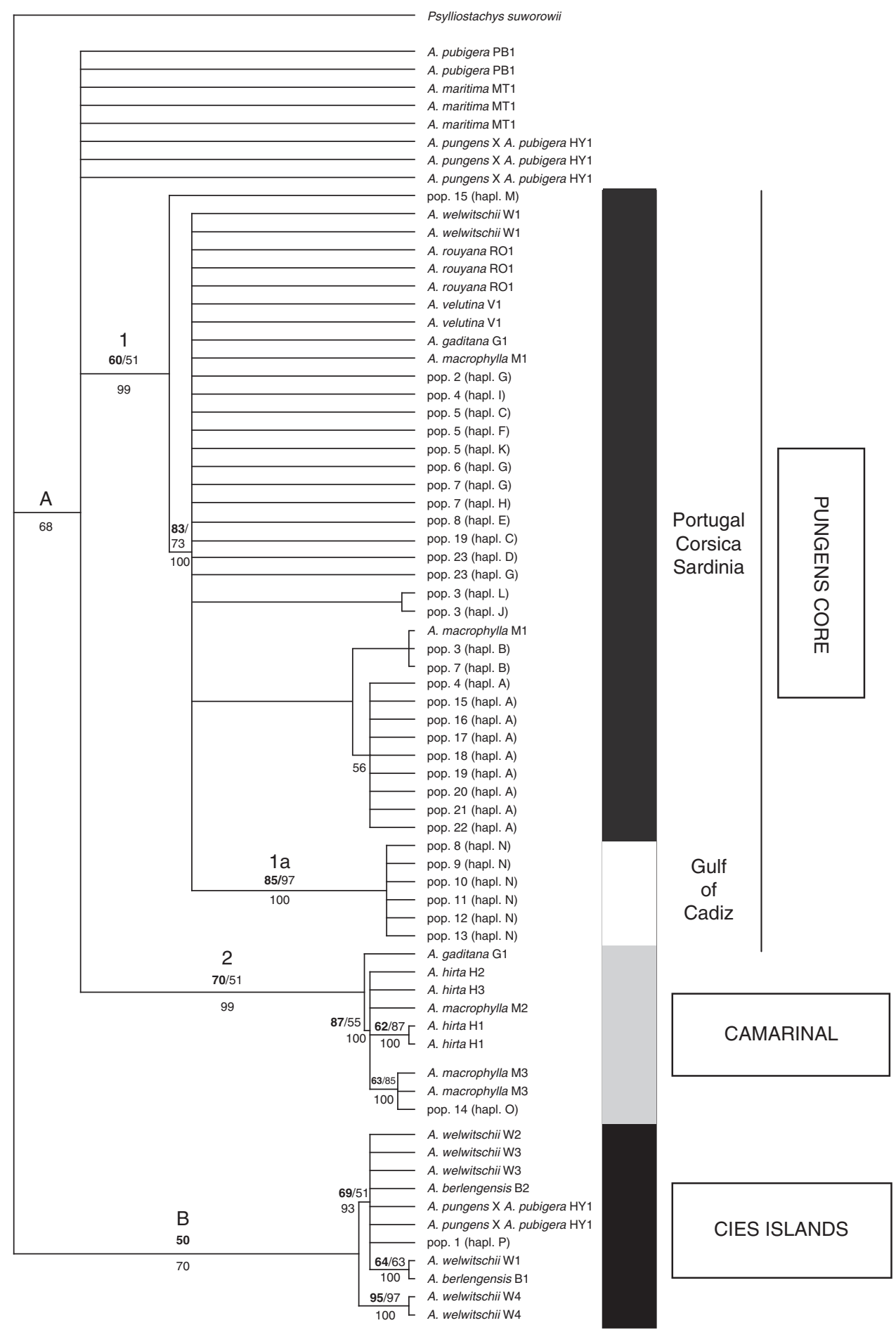

Figure 4 Chloroplast phylogenetic tree (trnL-F, trnS-fM, matK) of A. pungens and sympatric congeners. Overall, $50 \%$ majority rule consensus of $87 \mathrm{MP}$ trees depicting the phylogenetic relationships between the plastid haplotypes of 23 populations of A. pungens and 36 individuals from 19 populations of 10 coastal and subcoastal congeners. Bootstrap supports above $50 \%$ are indicated above the branches both without (in bold) and with an additional binary character representing the presence/absence of indels. Posterior probabilities of the Bayesian analysis are indicated below branches. The names of chloroplast haplotypes and main genetic groups in A. pungens are indicated according to Figures $2 \mathrm{a}$ and $\mathrm{b}$. A. pungens individuals are labeled with population numbers as described in Supplementary information S1 and Figure 1a. The name and population of origin of the other species follow Supplementary information S2 and Figure 1b.

biparentally inherited ITS markers was detected. Although plastid sequences revealed an exclusive haplotype (P; Figures $2 \mathrm{a}$ and $\mathrm{b}$ ) that was remarkably divergent from the rest of the populations, the ITS data indicated similarity with the Portuguese-CorsoSardinian populations of $A$. pungens (Figure 2c). This is revealed by the sharing of the $\mathrm{R} 1 \mathrm{~b}$ profile with the closest mainland population (population 2-Albufeira), 
Sardinia (populations 19-23) and two Corsican individuals (population 16). The presence of ciliate leaves related the Cíes population to the PortugueseCorso-Sardinian genetic group (Figure 3), in agreement with the ITS.

The sequencing of plastid markers in the sympatric and parapatric species provided a picture of how these congeners relate to the anomalous disjunct northernmost and southernmost populations of $A$. pungens (Figures 2a and $b$ ). In the plastid phylogeny, $A$. pungens from the Cíes islands (population 1; clade B; Figure 4) appeared to be closer to $A$. berlengensis (populations B1 and B2) and $A$. welwitschii (populations $\mathrm{W} 1, \mathrm{~W} 2$ and $\mathrm{W} 3$ ) than to mainland A. pungens (populations 2-14; clade A; Figure 4). Similarly, $A$. pungens from Camarinal (population 14) was placed in clade 2 together with its sympatric southern congeners A. gaditana, A. hirta and A. macrophylla, apart from all other populations of the species. The latter relationship was also supported by the sharing of the ITS R1e profile that involved two ITS polymorphic sites at positions 380 and 448 (Table 1) with $A$. macrophylla.

The other species from Cíes, $A$. pubigera and $A$. maritima, were part of the polytomy in clade 1 in the plastid phylogeny (Figure 4). Moreover, in the Cíes islands, the five putative $A$. pungens $\times A$. pubigera hybrid individuals studied were shown to fall either close to the plastid haplotype $\mathrm{P}$ of $A$. pungens (two individuals) or close to $A$. pubigera (three individuals in a polytomy with the four major clades). Finally, these hybrids showed additive ITS polymorphic sites for all five parsimonyinformative positions differing between the typical $A$. pungens ribotype $\mathrm{R} 1$ and the typical $A$. pubigera $\mathrm{R} 7$ (Table 1).

\section{Discussion}

Plastid DNA and ITS data of A. pungens show clear footprints of colonization and hybridization.

\section{Colonization of Corsica-Sardinia and the Gulf of Cadiz}

Previous AFLP data suggest that the Atlantic-Mediterranean disjunction of $A$. pungens resulted from the longdistance dispersal of Portuguese plants into CorsicaSardinia (Piñeiro et al., 2007). These data are supported by the detection in Corsica and Sardinia of the typical western Iberian Armeria ITS ribotype (R1a without additions with respect to R5), the presence of plastid haplotypes also found in Portugal and the morphological similarity with Portuguese populations (no differences in quantitative characters and the presence of ciliate leaves in both areas). In agreement with this scenario, the Portuguese source area exhibits a high number of plastid DNA haplotypes (10 of 16) within the same lineage, with almost identical values of $\mathrm{G}_{\text {st }}$ and $\mathrm{N}_{\text {st }}$ (Table 2), which suggests the long-term stability of these populations. No evidence for introgression from sympatric species accounting for such high plastid diversity levels in Portugal was apparent from the described pattern, even in Bordeira (population 6), which showed plastid DNA and ITS variants typical of the Portuguese lineage of $A$. pungens despite exhibiting unusually densely pubescent leaves and scapes.

A few individuals from several congeners (A. welwitschii, A. rouyana, A. velutina, A. macrophylla and
A. gaditana) showed plastid sequences closely related to those of $A$. pungens in Portugal (Figure 4; clade 1), suggesting either some incomplete sorting of ancestral polymorphisms or introgression of A. pungens into these populations. The fact that these closely related haplotypes occur in geographically distant populations makes the hypothesis of sorting of ancestral polymorphisms more likely. An extended population-level sample of chloroplast and nuclear markers covering the entire range of all the species may help to test this hypothesis.

Unlike previous AFLP markers, plastid sequences allowed the reconstruction of genealogical relationships between the distinct Gulf of Cadiz lineage and the remaining populations of the species. This lineage was revealed to be linked to southern Portuguese haplotypes and exhibited extremely low plastid diversity and lack of differentiation among populations. This genetic structure indicates that the Gulf of Cadiz lineage has recently colonized its current distributional area. In this context, genetic drift might have been the dominant evolutionary force, which might explain the possible extinction of the intermediate haplotypes between $\mathrm{E}$ and $\mathrm{N}$. The attainment of such levels of differentiation through long-term isolation seems less plausible, given the lack of correlation between haplotypes and geographical locations in the Gulf of Cadiz. Still, these levels might also be attained if a regional population extinction event was followed by recolonization from, for example, remnant populations in the east near the Gibraltar strait. On the other hand, differentiation of the Gulf Cadiz lineage by means of introgression from an extinct (or not sampled) lineage of a sympatric species also seems unlikely, given the notable genetic connection to the haplotypes found in A. pungens from Southern Portugal. In addition to their different genetic composition, the plants of the Gulf of Cadiz tend to exhibit glabrous to subglabrous leaves. Interestingly, classic taxonomic treatments had described only the dense pubescent populations that we found in Bordeira (Bernis, 1955; Pinto da Silva, 1972) but not the glabrous populations occurring along the Gulf of Cadiz.

The comparison between the plastid genetic structure of A. pungens in Corsica-Sardinia and in the Gulf of Cadiz revealed dissimilar genetic consequences of colonization in the two areas. In the Gulf of Cadiz, the loss of genetic diversity was associated with a significant change in the haplotype composition and frequencies. In contrast, the loss of plastid diversity observed in CorsicaSardinia was not accompanied by divergence from the Portuguese source populations. Therefore, the genetic founder effect was found to be more severe in the Gulf of Cadiz, where climatic conditions differ from the remaining populations, as compared with Corsica-Sardinia, where the probability of dispersal from Portugal is reduced by the much-larger physical distance (Piñeiro et al., 2007). This might indicate an important role of the establishment phase and natural selection during colonization. Nonetheless, to establish causal relationships between genotypes and ecological variables, more appropriate methods than the mere correlation between the neutral genetic variation and environmental conditions should be used. Future studies might include manipulative experiments to assess particular environmental factors as well as identification and survey of loci under selection (Karrenberg and Widmer, 2008). 
Hybridization in peripheral populations

The genetic pattern of the northernmost disjunct population on the Cíes islands shows inconsistency between the typical A. pungens morphology (ciliate leaves) and nuclear markers (ITS and AFLP; Piñeiro et al., 2007) on one side and the divergent maternally inherited plastid DNA on the other. This matches other cases of chloroplast capture following ancient hybridization (Rieseberg and Wendel, 1993; Arnold, 1997). The additional finding of plastid-haplotype sharing across species allows the rejection of the hypothesis of plastid DNA divergence through the isolation of $A$. pungens in the Cíes Islands and suggests introgression from the Portuguese coastal species $A$. welwitschii and $A$. berlengensis, or a related taxon. The habitat similarities of $A$. pungens and A. welwitschii (sandy coastal environments), in the context of the frequent interspecific hybridization in Armeria and the dynamic history of Quaternary vegetation in Europe, make it conceivable that both species showed different latitudinal boundaries in the past that favored contacts. Although the possibility that this plastid haplotype has persisted on the Cíes islands as a result of the random sorting of ancestral polymorphisms is less likely, it cannot be completely ruled out.

More straightforward is the interpretation of the origin of the plants in the Cíes islands occurring in the ecotone between sand dunes where $A$. pungens occurs and in granite cliffs where $A$. pubigera occurs. The detection of plastid haplotypes of the two species and additive ITS patterns in these individuals indicates that they may be part of a hybrid zone. Morphometric studies confirm the occurrence of such a hybrid zone containing different hybrid genotypes (Piñeiro R, Fuertes Aguilar J and Nieto Feliner G, unpublished data).

A. pungens in the disjunct population of the Cíes islands thus seems to have experienced at least two hybridization events at different times: past introgression from $A$. welwitschii, $A$. berlengensis or a closely related taxon and ongoing hybridization with $A$. pubigera. Whereas in the latter event an intermediate genetic pattern between the two parental species is still easily recognized, in the former event, the genetic traces of hybridization may have been partially erased by backcrossing toward $A$. pungens following patterns detected in the ITS and morphology. In plants, nuclear markers are frequently less introgressed and thus more congruent with morphology than plastid markers (Rieseberg and Wendel, 1993). This observation is attributed to the faster mutation rates and higher effective population size of the nuclear genome, although the effective migration of nuclear genes by pollen dispersal may be also implicated (Petit and Excoffier, 2009).

In Camarinal, recent introgression from $A$. macrophylla can also be easily inferred from the sharing of ITS polymorphisms and puberulous leaves, as well as the close relationship of plastid haplotypes. The habitat of this population (low open shrubs on fossil dunes) also supports introgression because it is intermediate between that of A.pungens (coastal sand dunes) and that of A. macrophylla (pine wood understory on sandy soils).

The observation of introgressed genotypes exclusively in the northern and southern marginal populations of $A$. pungens, despite the regular occurrence of sympatric species of Armeria along the Iberian coast, indicates that hybridization is favored in the margins of the distribu- tion. This pattern may be due to selection for introgressed genotypes at the latitudinal extremes of the species' range, which may allow the colonization of novel niches (Anderson and Stebbins, 1954; Abbott et al., 2003; Ellstrand, 2003). Alternatively, population dynamics in peripheral populations, characterized by reduced densities, may have favored the surfing of alleles from sympatric species in demographic equilibrium into A. pungens (Klopfstein et al., 2006, Petit and Excoffier, 2009).

\section{Conflict of interest}

The authors declare no conflict of interest

\section{Acknowledgements}

We are grateful to Carmen Rotenbühler and members of Alex Widmer's lab for help and support to RP during her stay in Zürich, to Christian Parisod and Rafael Hernandez for help in data analysis, to Laura Barrios for advice on statistical matters, to François Boillot (Conservatoire Botanique National Méditerranéen de Porquerolles) and Sébastien Gatelier (Estación Biológica de Doñana) for providing seeds, to Pedro Escobar for fieldwork support and to three anonymous reviewers for valuable comments on the paper. This work was supported by the Spanish Dirección General de Enseñanza Superior e Investigación Científica (Grant no. BOS2001-1839).

\section{References}

Abbott RJ (1992). Plant invasions, interspecific hybridization and the evolution of new plant taxa. Trends Ecol Evol 7: 401-405.

Abbott CL, Double MC (2003). Genetic structure, conservation genetics and evidence of speciation by range expansion in shy and white-capped albatrosses. Mol Ecol 12: 2953-2962.

Abbott RJ, James JK, Milne RI, Gillies ACM (2003). Plant introductions, hybridization and gene flow. Philos $T R$ Soc $B$ 358: $1123-1132$.

Albert V, Jonsson B, Bernatchez L (2006). Natural hybrids in Atlantic eels (Anguilla anguilla, Arostrata): evidence for successful reproduction and fluctuating abundance in space and time. Mol Ecol 15: 1903-1916.

Alsos I, Bronken Eidesen P, Ehrich D, Skrede I, Westergaard K, Jacobsen GH et al. (2007). Frequent long-distance plant colonization in the changing Arctic. Science 316: 1606-1609.

Álvarez I, Wendel JF (2003). Ribosomal ITS sequences and plant phylogenetic inference. Mol Phylogenet Evol 29: 417-434.

Anderson E, Stebbins JR (1954). Hybridization as an evolutionary stimulus. Evolution 8: 288-378.

Arnold ML (1997). Natural Hybridization and Evolution. Oxford University Press: Oxford.

Arrigoni PV (1970). Contributto alla conoscenza delle Armerie sardo-corse. Webbia 25: 137-182.

Baker HG (1966). The evolution, functioning and breakdown of heteromorphic incompatibility systems, I The Plumbaginaceae. Evolution 20: 349-368.

Baker A, Moeed A (1987). Rapid genetic differentiation and founder effect in colonizing populations of common mynas Acridotheres tristis. Evolution 41: 525-538.

Baldwin BG, Sanderson MJ, Porter JM, Wojciechowski MF, Campbell CS, Donoghue MJ (1995). The ITS region of nuclear ribosomal DNA: a valuable source of evidence on angiosperm phylogeny. Ann Mo Bot Gard 82: 247-277.

Barker JS, Frydenberg J, González J, Davies HI, Ruiz A, Sørensen JG et al. (2009). Bottlenecks, population differentiation 
and apparent selection at microsatellite loci in Australian Drosophila buzzatii. Heredity 102: 340-389.

Bernis F (1955). Revisión del género Armeria Willd. con especial referencia a los grupos ibéricos. Parte segunda (descriptiva de los grupos ibéricos). Anales Inst Bot Cavanilles 12: 77-252.

Choler P, Erschbamer B, Tribsch A, Gielly L, Taberlet P (2004). Genetic introgression as a potential to widen a species' niche: insights from alpine Carex curvula. Proc Natl Acad Sci USA 101: 171-176.

Clegg SM, Degnan SM, Kikkawa J, Moritz C, Estoup A, Owens IP (2002). Genetic consequences of sequential founder events by an island-colonizing bird. Proc Natl Acad Sci USA 99: 8127-8132.

Clegg M, Gaut B, Learn Jr G, Morton B (1994). Rates and patterns of chloroplast DNA evolution. Proc Natl Acad Sci USA 91: 6795-6801.

Clement M, Posada D, Crandall KA (2000). TCS: a computer program to estimate gene genealogies. Mol Ecol 9: 1657-1659.

Demesure B, Sodzi N, Petit R (1995). A set of universal primers for amplification of polymorphic non-coding regions of mitochondrial and chloroplast DNA in plants. Mol Ecol 4: 129-131.

Ellstrand NC (2003). Current knowledge of gene flow in plants: implications for transgene flow. Philos $T R$ Soc $B$ 358: 1163-1170.

Ellstrand NC, Schierenbeck KA (2000). Hybridization as a stimulus for the evolution of invasiveness in plants? Proc Nat Acad Sci USA 97: 7043-7050.

El Mousadik A, Petit R (1996). High level of genetic differentiation for allelic richness among populations of the argan tree [Argania spinosa (L Skeels] endemic to Morocco. Theor Appl Genet 92: 832-839.

Fuertes Aguilar J, Nieto Feliner G (2003). Additive polymorphisms and reticulation in an ITS phylogeny of thrifts (Armeria, Plumbaginaceae). Mol Phylogenet Evol 28: 430-447.

Fuertes Aguilar J, Rosselló JA, Nieto Feliner G (1999a). Nuclear ribosomal DNA (nrDNA) concerted evolution in natural and artificial hybrids of Armeria (Plumbaginaceae). Mol Ecol 8: 1341-1346.

Fuertes Aguilar J, Rosselló JA, Nieto Feliner G (1999b). Molecular evidence for the compilospecies model of reticulate evolution in Armeria (Plumbaginaceae). Systematic Biol 48: 735-754.

Goudet J (2001). FSTAT, a Program to Estimate and Test Gene Diversities and Fixation Indices Available from http:// wwwl.unil.ch/izea/softwares/fstat.html. Updated from Goudet (1995).

Grant PR, Grant BR, Petren K (2001). A population founded by a single pair of individuals: establishment, expansion, and evolution. Genetica 112-113: 359-382.

Gutiérrez Larena B, Fuertes Aguilar J, Nieto Feliner G (2002). Glacial-induced altitudinal migrations in Armeria (Plumbaginaceae) inferred from patterns of chloroplast DNA haplotype sharing. Mol Ecol 11: 1965-1974.

Hall TA (1999). BioEdit: a user-friendly biological sequence alignment editor and analysis. Nucleic Acids Symp Ser 41: 95-98.

Karrenberg S, Widmer A (2008). Ecologically relevant genetic variation from a non-arabidopsis perspective. Current Opin Plant Biol 11: 156-162.

Kelchner SA (2000). The evolution of non-coding chloroplast DNA and its application in plant systematics. Annals Mo Bot Gard 87: 482-498.

Keller I, Veltsos P, Nichols RA (2008). The frequency of rDNA variants within individuals provides evidence of population history and gene flow across a grasshopper hybrid zone. Evolution 62: 833-844.

Klopfstein S, Currat M, Excoffier L (2006). The fate of mutations surfing on the wave of a range expansion. Mol Biol Evol 23: 482-490.
Lavergne S, Molofsky J (2007). From the cover: increased genetic variation and evolutionary potential drive the success of an invasive grass. Proc Natl Acad Sci USA 104: 3883-3888.

Lefèbvre C (1974). Relations génétiques entre des populations d'Armeria maritima (Mill.) Willd. CR Acad Sci Paris, Sciences de la vie/Life Sciences 278: 1573-1576.

Lihová J, Fuertes Aguilar J, Marhold K, Nieto Feliner G (2004). Origin of the disjunct tetraploid Cardamine amporitana (Brassicaceae) assessed with nuclear and chloroplast DNA sequence data. Am J Bot 91: 1231-1242.

Lledó MD, Crespo MB, Cameron KM, Fay MF, Chase MW (1998). Systematics of Plumbaginaceae based upon cladistic analysis of rbcL sequence data. Syst Bot 23: 21-29.

Mayr E (1942). Systematics and the Origin of Species. Columbia University Press: New York.

Moody ML, Les DH (2002). Evidence of hybridity in invasive watermilfoil (Myriophyllum) populations. Proc Nat Acad Sci USA 99: 14867-14871.

Nieto Feliner G (1990). Armeria. In: Castroviejo S, Laínz M, López G, et al. (eds). Flora Iberica. Consejo Superior de Investigaciones Científicas: Madrid. pp 642-721.

Nieto Feliner G, Fuertes Aguilar J, Rosselló JA (2001). Can extensive reticulation and concerted evolution result in a cladistically structured molecular data set? Cladistics 17: 301-312.

Nieto Feliner G, Gutiérrez Larena B, Fuertes Aguilar J (2004). Fine scale geographical structure, intra-individual polymorphism and recombination in nuclear ribosomal internal transcribed spacers in Armeria (Plumbaginaceae). Ann Bot 93: 189-200.

Nieto Feliner G, Rosselló JA (2007). Better the devil you know? Guidelines for insightful utilization of nrDNA ITS in specieslevel evolutionary studies in plants. Mol Phylogenet Evol 44: 911-919.

Nylander JAA (2003). MrModeltest 1.0b. A Simplified Version of David Posada's 'Modeltest 3.06'. Department of Systematic Zoology: Uppsala, Sweden http://www ebc.uu.se/systzoo/ staff/nylander.html.

Petit RJ, Bodenes C, Ducousso A, Roussel G, Kremer A (2004). Hybridization as a mechanism of invasion in oaks. New Phytol 161: 151-164.

Petit RJ, Duminil J, Fineschi S, Hampe A, Salvini D, Vendramin GG (2005). Comparative organization of chloroplast, mitochondrial and nuclear diversity in plant populations. Mol Ecol 14: 689-701.

Petit RJ, Excoffier L (2009). Gene flow and species delimitation. Trends Ecol Evol 24: 386-393.

Pinto da Silva R (1972). Armeria Willd. In: Tutin TG, Heywood VH, Burges NA, et al. (eds). Flora Europaea. Cambridge University Press: Cambridge, New York, Vol. 3.

Piñeiro R, Costa A, Fuertes Aguilar J, Nieto Feliner G (2009). Overcoming paralogy and incomplete lineage sorting to detect a phylogeographic signal: a GapC study of Armeria pungens. Botany 87: 164-177.

Piñeiro R, Fuertes Aguilar J, Draper Munt D, Nieto Feliner G (2007). Ecology matters: Atlantic-Mediterranean disjunction in the sand-dune shrub Armeria pungens (Plumbaginaceae). Mol Ecol 16: 2155-2171.

Pons O, Petit RJ (1996). Measuring and testing genetic differentiation with ordered versus unordered alleles. Genetics 144: 1237-1245.

Pruett CL, Winker K (2005). Northwestern song sparrow populations show genetic effects of sequential colonization. Mol Ecol 14: 1421-1434.

Rieseberg LH, Wendel JF (1993). Introgression and its consequences in plants. In: Harrison R (ed). Hybrid Zones and the Evolutionary Process. Oxford University Press: New York. pp 70-109. 
Ronquist F, Huelsenbeck JP (2003). MRBAYES 3: Bayesian phylogenetic inference under mixed models. Bioinformatics 19: $1572-1574$.

Rozas J, Sánchez-DelBarrio JC, Messeger S, Rozas R (2003). DNA polymorphisms analysis by the coalescent and other methods. Bioinformatics 19: 2496-2497.

Rozen S, Skaletsky H (2000). Primer3 on the WWW for general users and for biologist programmers. In: Krawetz S, Misener S (eds). Bioinformatics Methods and Protocols: Methods in Molecular Biology. Humana Press: Totowa, NJ. pp 365-386.

Swofford D (2002). Phylogenetic Analysis Using Parsimony (and other Methods), Version 4 Sinauer Associates: Sunderland, MA.
Taberlet P, Gielly L, Pautou G, Bouvet J (1991). Universal primers for amplification of three non-coding regions of chloroplast DNA. Plant Mol Biol 17: 1105-1109.

Tauleigne-Gomes C, Lefèbvre C (2005). Natural hybridization between two coastal endemic species of Armeria (Plumbaginaceae) from Portugal. 1. Populational in situ investigations. Plant Syst Evol 250: 215-230.

Tiébré M-S, Vanderhoeven S, Saad L, Mahy G (2007). Hybridization and sexual reproduction in the invasive alien Fallopia (Polygonaceae) complex in Belgium. Ann Bot 99: 193-203.

Tregenza T (2002). Divergence and reproductive isolation in the early stages of speciation. Genetica 116: 291-300.

Supplementary Information accompanies the paper on Heredity website (http://www.nature.com/hdy) 